\title{
Enfoque minimalista en el implante de válvula aórtica percutánea
}

\author{
René Hameau ${ }^{1}$, Nicolás Veas ${ }^{2}$, José Luis Winter ${ }^{2}$, Martín Valdebenito ${ }^{2}$, Rodrigo Muñoz ${ }^{2}$, Alberto Fuensalida ${ }^{1}$, Dante Lindefjeld. ${ }^{2}$ \\ 1. Residente Cardiologia Intervencional, Hospital Sótero del Río/Pontificia Universidad Católica de Chile, Santiago, Chile. \\ 2. Cardiólogo Intervencionista, Unidad de Cardiología Intervencional y Hemodinamia, Hospital Sótero del Río, Santiago, Chile.
}

Introducción: En pacientes con estenosis Aórtica (EA) severa sintomática, el implante de válvula aórtica percutánea transcatéter (TAVI) por vía transfemoral constituye el estándar de tratamiento en aquellos de riesgo quirúrgico intermedio o alto. El uso de un abordaje minimalista ha demostrado ser seguro y efectivo, si bien no existen reportes sobre la realidad nacional

Métodos: Estudio descriptivo sobre la experiencia con pacientes sometidos al implante de TAVI bajo un protocolo minimalista en Unidad de Cardiología Intervencional y Hemodinamia del Hospital Sótero del Río desde Enero de 2018. Se analizaron las variables clínicas de los pacientes y del procedimiento así como desenlaces clínicos intrahospitalarios y seguimiento alejado.

Resultados: Entre Enero 2018 hasta Abril 2019, un total de 10 pacientes fueron sometidos al implante de
TAVI por vía transfemoral. El score STS-PROM promedio fue de 7,1. Se logró un implante exitoso en el $100 \%$ de los casos con un gradiente medio residual de $8 \mathrm{mmHg}$ y sin leak moderado a severo en ningún paciente. No hubo eventos cerebrovasculares isquémicos perioperatorios ni muerte en este grupo. Se requirió implante de marcapasos definitivo en 3 pacientes y un paciente presentó hematoma femoral perioperatorio que requirió transfusión de glóbulos rojos. La mediana de la estadía hospitalaria fue de 2 días.

Conclusiones: El uso de una estrategia minimalista para el implante de TAVI en nuestra realidad nacional es seguro y aplicable. Los resultados perioperatorios y a 30 días fueron comparables a los descritos en experiencias internacionales.

Palabras Clave: TAVI, minimalismo, estenosis aórtica.

\section{Correspondencia:}

Dr. Dante Lindefjeld C.

Unidad de Cardiología Intervencional y Hemodinamia.

Hospital Sótero del Río.

dslindefjeld@gmail.com 


\section{A minimalist approach for transcatheter aortic valve replacement}

Background: In patients with symptomatic severe aortic stenosis, transcatheter percutaneous aortic valve implant (TAVI) is the standard treatment in those with intermediate or high surgical risk. The use of a minimalist approach has proven to be safe and effective, although there are no reports on the national reality

Methods: Descriptive study on the experience with patients undergoing TAVI implantation under a minimalist protocol at the Interventional Cardiology and Hemodynamics Unit of the Hospital Sótero del Río since January 2018. Clinical characteristics of the patients and the procedure were analyzed as well as intrahospital outcomes and at 30-days follow up. Results: Between January 2018 and April 2019, a total of 10 patients underwent TAVI implantation by transfemoral approach in our institution. The average STS-PROM score was 7.1. A successful implant was achieved in $100 \%$ of cases with an average residual gradient of $8 \mathrm{mmHg}$ and no moderate to severe leak in any patient. There were no perioperative ischemic cerebrovascular events nor death in this group. A definitive pacemaker implant was required in 3 patients and one patient developed femoral hematoma that required red blood cell transfusion. The median hospital stay was 2 days.

Conclusions: The use of a minimalist strategy for TAVI implantation in our national reality is safe and applicable. Immediate results and at 30-days follow up were comparable to those described in international experiences.

Key Words: TAVI, minimalist, aortic stenosis. 


\section{Introducción:}

La estenosis aórtica (EA) es la valvulopatía primaria que con mayor frecuencia requiere de tratamiento quirúrgico o percutáneo tanto en Europa como en EEUU ${ }^{1}$ con un espectro de indicaciones que sigue aumentando. El implante percutáneo de válvula aórtica (TAVI) es una terapia ampliamente difundida pero existen aspectos de la técnica, así como del manejo posterior de estos pacientes, que presentan alta variabilidad entre los distintos centros y que aún permanecen como objeto de debate. Elementos como el tipo de sedación, el rol del marcapasos transitorio, la rehabilitación postoperatoria o los criterios de alta siguen siendo motivo de estudio en el ámbito de la cardiología intervencional ${ }^{2}$. Por otra parte, existe una búsqueda constante para tratar de optimizar el uso de recursos, disminuyendo las complicaciones y acortando la estadía hospitalaria.

En Chile se observa un progresivo aumento en el uso de técnicas minimalistas para el implante, sin casuísticas nacionales publicadas. El objetivo de este estudio es reportar los resultados iniciales de una serie de casos de implante de TAVI bajo un protocolo minimalista en la Unidad de Cardiología Intervencional y Hemodinamia del Hospital Sótero del Río.

\section{Material y Métodos:}

Estudio descriptivo de serie de casos, sobre pacientes sometidos al implante de TAVI bajo un protocolo minimalista en la Unidad de Cardiología Intervencional y Hemodinamia del Hospital Sótero del Río desde Enero 2018 a Abril 2019.

\section{Los criterios de inclusión fueron:}

1) Hombre o mujeres $>65$ años y portadores de estenosis aórtica severa sintomática.

2) Acceso transfemoral adecuado.

3) Pacientes considerados de alto riesgo quirúrgico o inoperables tras la discusión con el equipo de Cirugía Cardíaca (Heart team).

Se excluyó a pacientes con aorta bicúspide, aquellos con indicación de otra cirugía cardiaca por enfermedad coronaria o valvulopatía concomitante.

Una vez confirmada la indicación de TAVI, se aplicó un protocolo de 4 componentes:

1.- Peri procedimiento: Previo la TAVI los pacientes fueron evaluados clínicamente y con el uso de ecocardiograma, coronariografía y Angio-Tomografía computada (AngioTAC) para la planificación del procedimiento. El implante se realizó en el Laboratorio de Hemodinamia bajo anestesia local y sedación consciente, sólo con apoyo de Ecocardiografía transtorácica (ETT).

Con el uso de Fentanilo y Midazolam se buscó una ansiolisis suave o sedación consciente con el objetivo de mantener siempre un contacto verbal entre el operador y el paciente. El acceso femoral fue pre-cerrado con 2 dispositivos Proglide Perclose (Abbott Vascular ${ }^{\circledR}$ ) + un acceso femoral contralateral para angiografía con pigtail 5F. Se realizó protección femoral contralateral en aquellos pacientes con enfermedad vascular periférica significativa y que, a juicio del operador, hicieran prever un riesgo elevado de lesión vascular (disección, perforación, etc.), u oclusión aguda. Se instaló una sonda marcapasos transitoria vía venosa femoral y apoyo con drogas vasoactivas por vía periférica para mantener una hemodinamia estable cuando fuese requerido.

Se utilizaron válvulas de tipo autoexpandibles: Evolut ${ }^{\circledR}$ o Evolut Pro (Medtronic, Minnesota ${ }^{\circledR}$, USA), según disponibilidad y se implantaron siguiendo las recomendaciones del fabricante.

Se consideró un implante exitoso aquel que cumpliera los criterios VARC23: ausencia de mortalidad + adecuada posición anatómica del implante + correcto funcionamiento (sin "mismatch" prótesis-paciente, gradiente medio $<20 \mathrm{mmHg}$ y ausencia de leak moderado a severo).

El equipo a cargo del procedimiento consistió en: dos Cardiólogos Intervencionistas a cargo del implante, un Cardiólogo a cargo del apoyo con ETT y monitoreo hemodinámico, una Enfermera de pabellón para administración de medicamentos y monitoreo, un Tecnólogo médico para control del angiógrafo y una Técnico paramédico. (Figura 1). No se contó con la presencia de Cardiocirujano o Anestesista en pabellón pero ambos equipos se encontraban notificados previamente y con la posibilidad de acceder rápidamente a este en caso de emergencia.

2.- Postoperatorio inmediato: Retiro de todos los accesos vasculares centrales en pabellón utilizando los dispositivos de cierre. Posterior al procedimiento, los pacientes fueron trasladados a la Unidad Coronaria para monitorización. Si el paciente no presentaba trastornos del ritmo significativos (BCRI, Bloqueo AV de alto grado, Bradicardia extrema) dentro de las primeras 24 horas, se procedió al retiro de la sonda marcapasos transitoria, según lo recomendado por la literatura. ${ }^{4}$ 


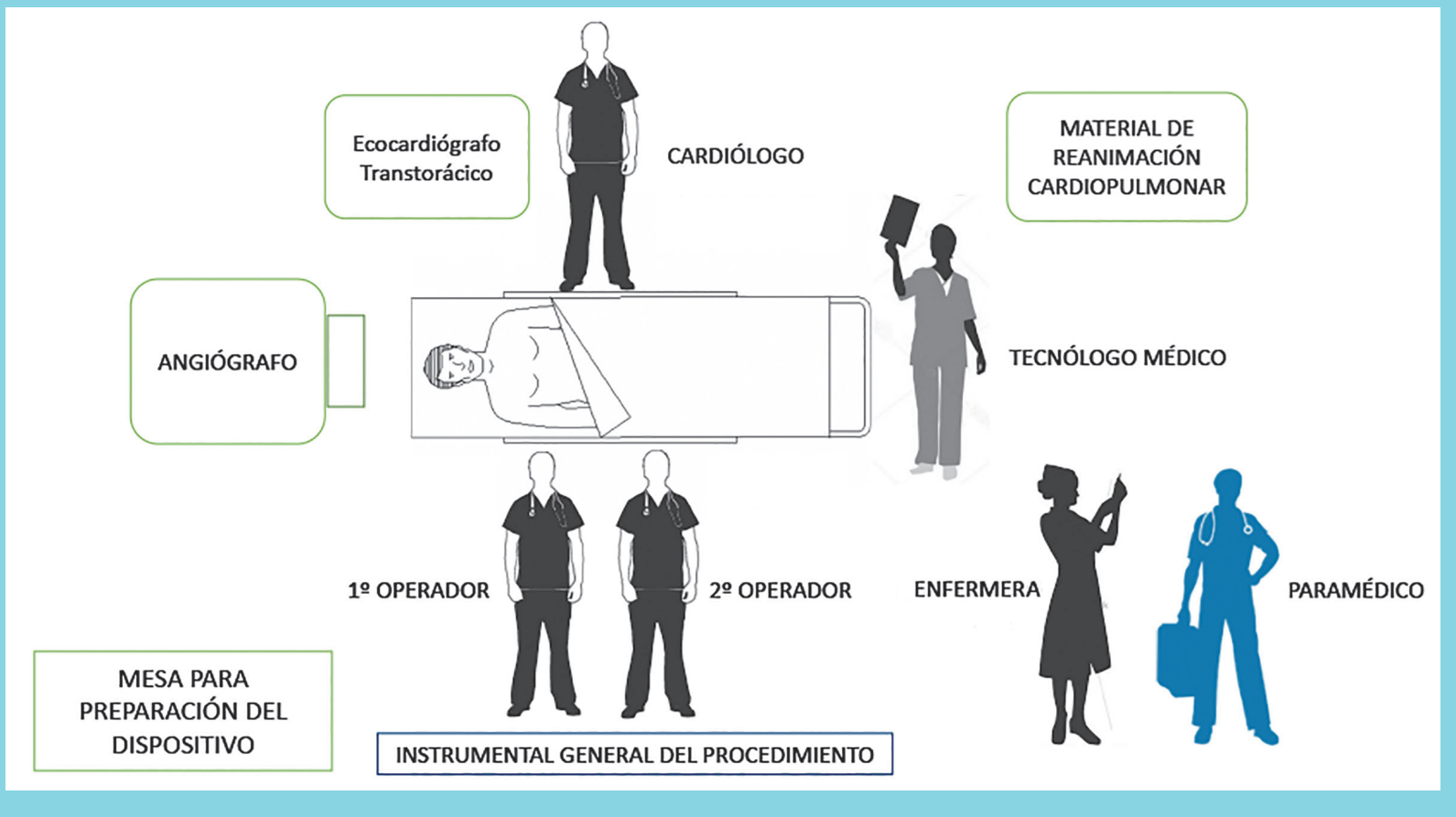

3.- Evaluación pre alta: Se realizó un ETT, ECG y exámenes de laboratorio. De acuerdo a la evolución clínica y deambulación precoz, se definía la eventual alta y se procedía a la educación del paciente/familiares así como el agendamiento del control ambulatorio.

4.- Seguimiento: Control ambulatorio en Policlínico de Cardiología a los 30 días.

El objetivo primario fue evaluar la seguridad de este protocolo en pacientes TAVI, valorando las complicaciones intraprocedimiento e intrahospitalarias (vasculares, tasa de marcapasos definitivo, leak para valvular), días de hospitalización y mortalidad a 30 días. Como objetivos secundarios se buscaba describir el perfil de pacientes sometidos a este protocolo, así como los detalles técnicos de cada intervención (Ej: duración del procedimiento, tipo de sedación utilizada y sus dosis, gradiente aórtico pre y post, dosis de radiación, etc).

\section{Análisis de datos:}

Mediante la revisión de los registros clínicos se caracterizaron las variables del procedimiento, eventos adversos, parámetros hemodinámicos así como ecocardiográficos, antecedentes clínicos y de laboratorio de cada sujeto. Para la descripción de variables cualitativas se empleó tablas de frecuencia. Las variables cuantitativas se describieron con la media \pm desviación estándar si su distribución era normal. En caso contrario, se utilizó mediana + valores min-max.

\section{Resultados:}

En el período entre Enero de 2018 y Abril de 2019, un total de 10 pacientes fueron rechazados para resolución quirúrgica y cumplieron los criterios de inclusión para el uso de este protocolo. La edad promedio fue de $79 \pm$ 4,9 años y un $70 \%$ de ellos era de sexo femenino.

Las comorbilidades más frecuentes fueron la HTA (80\%) y Dislipidemia (40\%). Un tercio de los pacientes padecía de enfermedad renal crónica (ERC) en hemodiálisis y un $20 \%$ tenía antecedentes de DM2. Un $40 \%$ tenía antecedentes de angioplastia coronaria y un $10 \%$ de cirugía de revascularización previa (Tabla 1). Los síntomas más frecuentes fueron la disnea de esfuerzo y la angina. Del total de pacientes, sólo 1 presentaba el antecedente de Fibrilación auricular y uso de terapia anticoagulante oral previa.

El tiempo promedio desde que se toma contacto con el equipo de Hemodinamia para la TAVI y se realiza el estudio coronario hasta el implante de la válvula fue de $315 \pm 217$ días. Sólo en 1 caso se realizó angioplas- 


\begin{tabular}{|c|c|c|c|c|c|c|c|c|c|c|c|}
\hline PACIENTE & EDAD: & SEXO: & HTA & DM & DLP & TABACO & IAM ANTIGUO: & $\begin{array}{c}\text { ERC EN } \\
\text { HEMODIALLISIS }\end{array}$ & $\begin{array}{l}\text { FEV } \\
\text { (\%) }\end{array}$ & $\begin{array}{c}\text { Gradiente } \\
\text { aortico medio } \\
\text { (mmHg) }\end{array}$ & STS SCORE \\
\hline 1 & 75 & M & $\mathrm{Si}$ & $\mathrm{Si}$ & No & No & $\mathrm{Si}$ & No & 68 & 40 & 7,09 \\
\hline 2 & 71 & $\mathrm{~F}$ & $\mathrm{Si}$ & No & No & No & No & Si & 55 & 41 & 5,90 \\
\hline 3 & 76 & $M$ & No & No & No & No & No & $\mathrm{Si}$ & 43 & 42 & 9,05 \\
\hline 4 & 87 & $\mathrm{~F}$ & $\mathrm{Si}$ & No & $\mathrm{Si}$ & No & $\mathrm{Si}$ & No & 54 & 68 & 5,30 \\
\hline 5 & 75 & $\mathrm{~F}$ & $\mathrm{Si}$ & No & $\mathrm{Si}$ & $\mathrm{Si}$ & No & No & 70 & 42 & 10,15 \\
\hline 6 & 75 & $\mathrm{~F}$ & $\mathrm{Si}$ & $\mathrm{Si}$ & No & No & No & No & 70 & 54 & 7,10 \\
\hline 7 & 82 & $M$ & $\mathrm{Si}$ & No & $\mathrm{Si}$ & $\mathrm{Si}$ & No & $\mathrm{Si}$ & 60 & 50 & 11,23 \\
\hline 8 & 82 & $\mathrm{~F}$ & $\mathrm{Si}$ & No & $\mathrm{Si}$ & No & No & No & 70 & 40 & 4,0 \\
\hline 9 & 84 & $\mathrm{~F}$ & No & No & No & No & No & No & 45 & 54 & 4,10 \\
\hline 10 & 83 & $\mathrm{~F}$ & $\mathrm{Si}$ & No & No & No & No & No & 66 & 53 & 7,07 \\
\hline
\end{tabular}

tía coronaria como preparación del procedimiento. No hubo revascularizaciones percutáneas concomitantes al implante de TAVI.

La mayoría de los pacientes se presentó con función sistólica preservada con una fracción de eyección del ventrículo izquierdo promedio de $60.1 \pm 6.1 \%$. El gradiente aórtico medio fue 48,2 $\pm 7.8 \mathrm{mmHg}$ y un área valvular de $0,65 \pm 0,18 \mathrm{~cm} 2$. En cuanto al riesgo quirúrgico, la mortalidad calculada a 30 días por score STSPROM fue $7,1 \%$, con un mínimo de 4 y un máximo de 11,2 puntos.

La totalidad de los implantes se realizó por acceso femoral derecho; asociado a uno femoral contralateral para la inserción de un pigtail 5F. Como apoyo imagenológico se utilizó únicamente ETT según el protocolo. La sedación consciente fue dirigida por el mismo equipo de Hemodinamia mediante la asociación de Fentanilo (promedio $68 \pm 31 \mathrm{ug}$ ) y Midazolam (promedio $1,7 \pm 0,7 \mathrm{mg}$ ). En ninguno de los casos fue necesaria la conversión a anestesia general. La duración promedio del procedimiento fue $109 \pm 36$ min y la dosis promedio de contraste utilizada fue de $215 \mathrm{ml}$. La dosis media de radiación fue de 1,83 Gy.

Un $50 \%$ de los implantes se realizó con la válvula autoexpandible Evolut ${ }^{\circledR}$ y un número equivalente con Evolut Pro. La mayor parte de los pacientes (60\%) re- cibió una válvula \#26, mientras que $10 \%$ requirieron una válvula \#23 y $30 \%$ una \#29. Se obtuvo un implante exitoso de la válvula en la totalidad de los casos, con un gradiente valvular medio post procedimiento por ecocardiograma pre alta de $8 \pm 2 \mathrm{mmHg}$ y ausencia de leak para valvular moderado-severo en todos ellos.

La mediana de hospitalización fue 2 días. Un paciente presentó una complicación no asociada al procedimiento (estenosis de arteria mesentérica conocida previamente y sepsis por traslocación bacteriana) que requirió resolución quirúrgica y que prolongó su estadía a 57 días.

La complicación más frecuente fue el implante de marcapasos definitivo en un $30 \%$ de los casos: 1 paciente a las 24 horas (Bradicardia sinusal persistente), otro a las 48 hrs (Bloqueo AV completo) y un tercero a los 23 días post implante (Bloqueo AV completo). De aquellos pacientes que requirieron un marcapasos definitivo, 2 tenían predictores previos: bradicardia sinusal en un caso y trastornos previos de la conducción intraventricular en el otro sujeto. Un caso (10\%) requirió de transfusión de glóbulos rojos por hematoma femoral perioperatorio desde el sitio de inserción venoso utilizado para la sonda marcapasos. No hubo muertes, accidente cerebrovascular $(\mathrm{ACV})$ ni falla renal aguda en el período intrahospitalario (Tabla 2). El 100\% de los pacientes 


\begin{tabular}{|c|c|c|c|c|c|c|c|c|c|c|}
\hline PACENTE & $\begin{array}{l}\text { DOSIS FENTANILO } \\
\text { (UB) }\end{array}$ & $\begin{array}{l}\text { DOSIS } \\
\text { MIDAZOLAM } \\
\text { (mg) }\end{array}$ & TIPO DE VÁLVULA & $\begin{array}{c}\text { DURACIÓN } \\
\text { ESTADIA } \\
\text { HOSPTTALARIA } \\
\text { (Dlas) }\end{array}$ & $\begin{array}{l}\text { IMPLANTE DE } \\
\text { MARCAPASOS } \\
\text { DEFINITIVO }\end{array}$ & $\begin{array}{l}\text { N2 DIAS DE } \\
\text { HOSPTALIZACIÓN }\end{array}$ & $\begin{array}{l}\text { COMPLICACIONES } \\
\text { VASCULARES } \\
\text { MAYORES }\end{array}$ & $\begin{array}{l}\text { MORTALIDAD } \\
30 \text { DIAS }\end{array}$ & ACVA 30 D/AS & $\begin{array}{l}\text { LEAK MODERADO } \\
\text { OSEVERO }\end{array}$ \\
\hline 1 & 75 & 1 & Evolut $R \# 26$ & 2 & No & 2 & No & No & No & No \\
\hline 2 & 30 & 0 & Evolut $R \# 26$ & 1 & $\begin{array}{l}\text { Si (A las } 24 \mathrm{hrs} \\
\text { post implante) }\end{array}$ & 1 & $\begin{array}{l}\text { Si (Hematoma } \\
\text { sitio punción } \\
\text { venoso) }\end{array}$ & No & No & No \\
\hline 3 & 100 & 2 & Evolut Pro \#29 & 1 & No & 1 & No & No & No & No \\
\hline 4 & 100 & 2 & Evolut Pro \#26 & 1 & No & 1 & No & No & No & No \\
\hline 5 & 75 & 2 & Evolut $R \# 26$ & 1 & No & 1 & No & No & No & No \\
\hline 6 & 50 & 2 & Evolut $R \# 26$ & 2 & No & 2 & No & No & No & No \\
\hline 7 & 50 & 2 & Evolut Pro \#26 & 57 & $\begin{array}{l}\text { Si (Implante a } \\
\text { los } 23 \text { dias post } \\
\text { implante) }\end{array}$ & 57 & No & No & No & No \\
\hline 8 & 125 & 2 & Evolut $R \# 23$ & 2 & No & 2 & No & No & No & No \\
\hline 9 & 50 & 3 & Evolut Pro $\# 29$ & 2 & No & 2 & No & No & No & No \\
\hline 10 & 25 & 1 & Evolut Pro $\# 29$ & 5 & $\begin{array}{l}\text { Si (Implante a } \\
\text { las } 48 \text { hrs) }\end{array}$ & 5 & No & No & No & No \\
\hline
\end{tabular}

ACV: Accidente cerebrovascular isquémico.

fue dado de alta bajo terapia antiplaquetaria dual. Sólo un paciente (10\%) desarrolló Fibrilación auricular en el seguimiento y cuya aparición ocurrió a los 14 días post implante (dentro del período intrahospitalario).

A los 30 días de seguimiento no se registró mortalidad; un $50 \%$ se encontraba en capacidad funcional I e igual porcentaje en capacidad funcional II.

\section{Discusión:}

Si bien en sus inicios, la TAVI se realizaba bajo anestesia general, el desarrollo de dispositivos de menor tamaño, la mayor experiencia de los operadores y la necesidad de disminuir los costos han hecho que muchos centros adopten estrategias llamadas "minimalistas" que incluyen, entre otros elementos, el uso de la sedación consciente y protocolos de alta precoz. Esta fue descrita por primera vez en Europa el año $2008^{5}$ y desde entonces existe un creciente interés por su desarrollo.

En los últimos años se ha demostrado beneficio de la sedo-analgesia versus anestesia general en pacientes sometidos a TAVI ${ }^{6}$. Se hace especialmente necesario en este tipo de pacientes, dado que el principal motivo para rechazar cirugía abierta, son los riesgos de la anestesia general, vía aérea difícil, ventilación mecánica prolongada y de difícil manejo. En nuestra institución el es- tándar es realizarlo sin anestesia general y un equipo de anestesia solo de apoyo. Esto nos facilita el procedimiento, sin cambiar nuestra forma habitual de trabajo. Con respecto al uso de Ecocardiograma transesofágico (ETE), los motivos para utilizarlos son, principalmente, posicionamiento de la válvula, relación con la válvula mitral, confirmar medidas valvulares, evaluar la insuficiencia valvular y para valvular post implante, excluir obstrucción coronaria y el diagnóstico precoz de derrame. Aunque todos esos puntos son muy razonables, actualmente con una buena planificación, nueva generación de dispositivos, ETT en sala y centros con experiencia en TAVI, el uso del ETE no es estrictamente necesario y no mejora los outcomes. En nuestro centro no se utiliza el ETE de forma rutinaria, lo cual nos ayuda a ser menos invasivos en casos complejos como estos, sin modificar los resultados del implante. Por lo anterior, se decide realizar con sedo-analgesia y sin ETE desde el inicio.

El principal cuestionamiento al uso de sedación consciente es la ausencia de estudios clínicos randomizados que validen sus beneficios en comparación con la anestesia general. A pesar de esto, existen reportes de experiencias favorables recientes. En 2019, el estudio Vancouver $3 \mathrm{M}^{7}$ reportó la experiencia de 411 pacientes 
en 13 centros de Canadá y EEUU en quienes se utilizó un enfoque minimalista similar al nuestro, aunque utilizando una prótesis balón-expandible. La mortalidad a 30 días fue de $2,9 \%$, logrando el alta al día siguiente en el $80 \%$ de los pacientes y dentro de 48 hrs en casi el $90 \%$. Las complicaciones vasculares ocurrieron en un 2,4\% y el implante de marcapasos definitivo en 5,7\%. No hubo casos de ACV invalidantes a 30 días. Otro aspecto destacable es que este protocolo fue aplicado en centros de alto, mediano y bajo volumen, sin observarse diferencias significativas en el desenlace primario.

En la misma línea, Ichibori $^{8}$ reporta el uso de sedación consciente incluso en pacientes con implantes urgentes de TAVI $(n=77)$, definidos como aquellos sujetos que recibieron la válvula en la misma hospitalización en que ingresaron por paro cardíaco, falla cardíaca severamente descompensada, síndrome coronario agudo o síncopes a repetición vs TAVI en pacientes electivos $(n=392)$. Al comparar ambos grupos a 30 días, no hubo diferencias en términos de ACV, infarto agudo al miocardio, complicaciones vasculares o mortalidad intrahospitalaria. Otras series recientes con la estrategia minimalista han reportado beneficios clínicos similares, así como una reducción de la estadía hospitalaria, lo que teóricamente pudiera disminuir los costos asociados. ${ }^{9}$

Por otro lado, sus detractores cuestionan estos reportes por los posibles sesgos de selección de pacientes, asignando a población menos enferma la estrategia minimalista o sesgos en cuanto a la terapia recibida considerando que probablemente aquellos operadores con mayor volumen estarán más abiertos a probar esta técnica. Otra limitación que plantean es la carencia del apoyo con ETE, recordando algunas series han encontrado asociación entre el uso de este y la reducción de eventos clínicos a largo plazo ${ }^{10}$. Mayr et al ${ }^{11}$ describen que aún en un centro de alto volumen, la incidencia de eventos adversos intra-pabellón bajo sedación consciente puede alcanzar un $10 \%$. El más frecuente fue la necesidad de conversión a anestesia general, principalmente por disconfort del paciente, seguido de distress respiratorio. Por lo anterior, este grupo aboga por la presencia un equipo de Anestesiología cada vez que se utilice esta estrategia.

El presente estudio reporta una serie de 10 casos consecutivos de implante de TAVI con un protocolo minimalista. Respecto a las características basales, la edad promedio de 79 años es similar a la reportada por estudios, como el NOTION ${ }^{12}$ o PARTNER $2^{13}$, pero destaca por poseer una proporción mayor de mujeres (70\%). En tér- minos de resultados, algunos registros sugieren que el sexo femenino tiene una mayor tendencia a complicaciones vasculares y hemorrágicas ${ }^{14}$, pero que a largo plazo este grupo tiene menor mortalidad que los hombres ${ }^{15}$, si bien estas diferencias han tendido a igualarse en los últimos años ${ }^{16}$.

La mayor parte de los pacientes poseía un riesgo quirúrgico intermedio, aunque también hubo sujetos de riesgo alto y cuya evolución fue similar en términos de sobrevida o riesgo de complicaciones intrahospitalarias.

El acceso femoral corresponde al abordaje de elección en la actualidad para el implante de TAVI, especialmente bajo la estrategia minimalista. Y pese a que las complicaciones del acceso son una de las más frecuentes del procedimiento, sólo 1 paciente presentó complicaciones vasculares que fueron manejadas de forma médica. La tasa de marcapasos aparentemente alta (30\%), se ve exagerada por el bajo número de pacientes que incluye esta serie. Además, debemos considerar que 2 de los sujetos ya poseían algunos predictores de la necesidad de marcapasos descritos en la literatura como: bloqueo completo de rama derecha, necesidad de implante profundo de la válvula, uso de prótesis autoexpandible, bloqueo AV de primer grado. El retiro precoz de la sonda de marcapaso transitoria, incluso antes de salir de pabellón o a las 24-48 horas, es una conducta descrita en la literatura y debe valorarse caso a caso en base a la presencia de predictores o aparición de trastornos eléctricos intra procedimiento. ${ }^{4}$

Un registro recientemente publicado por Pineda et al ${ }^{17}$ que incluyó a 47.546 pacientes sometidos a TAVI, mostró que la tasa de complicaciones que requirieron cirugía de rescate fue cercana a $1,17 \%$. Esto refuerza el concepto que, si bien debe existir una estrecha comunicación con el Equipo Cardioquirúrgico al momento del implante, su presencia física en el pabellón no es necesaria en todos los casos y debe ser valorada caso a caso. Las limitaciones de este estudio incluyen el bajo número de sujetos, además de ser un estudio en un solo centro. Por otro lado, únicamente utilizamos prótesis autoexpandibles y no en todos los pacientes se implantó una válvula de última generación.

Estudios nacionales ya han reportado la costo-efectividad de la TAVI vs la terapia médica conservadora ${ }^{18}$ y ante un sistema público de salud que debe equilibrar la aparición de nuevas técnicas con el uso eficiente de los recursos, esta estrategia puede constituir una alternativa interesante para el implante de TAVI. La demora de casi 10,3 meses entre la indicación de TAVI y su implante, 
nos lleva a reflexionar que siendo esta una opción no inferior al reemplazo quirúrgico, tanto en resultados clínicos como de costo-efectividad, es absolutamente necesario un desarrollo de políticas públicas que permitan el acceso oportuno a esta terapia.

Este estudio corresponde al primer reporte nacional so- bre el uso de una estrategia minimalista para el implante de TAVI y sugiere que este abordaje es seguro y aplicable en nuestro medio. Tanto la adecuada selección de pacientes, la experiencia del equipo de operadores y la existencia de un manejo protocolizado parecen ser claves en la obtención de resultados satisfactorios.

\section{Referencias}

1. NKOMO VT, GARDIN JM, SKELTON TN, GOTTDIENER JS, SCOTT CG, ENRIQUEZ-SARANO M. Burden of valvular heart diseases: a population-based study. Lancet. 2006;368(9540):1005-1011.

2. LESTER L, BRADY MB, BROWN CH. Sedation Versus General Anesthesia for TAVR: Where Do We Go From Here? J Cardiothorac Vasc Anesth. 2017;31(6):2055-2057.

3. PIETER KAPPETEIN A, HEAD SJ, GÉNÉREUX P, et al. Updated standardized endpoint definitions for transcatheter aortic valve implantation: The Valve Academic Research Consortium-2 consensus document. Eur Heart J. 2012;33:2403-2418.

4. AUFFRET V, PURI R, URENA M, et al. Conduction disturbances after transcatheter aortic valve replacement: Current status and future perspectives. Circulation. 2017;136:1049-1069.

5. BEHAN M, HAWORTH P, HUTCHINSON N, TRIVEDI U, LABORDE JC, HILDICK-SMITH D. Percutaneous aortic valve implants under sedation: Our initial experience. Catheter Cardiovasc Interv. 2008;72(7):1012-1015.

6. VILLABLANCA PA, MOHANANEY D, NIKOLIC K, et al. Comparison of local versus general anesthesia in patients undergoing transcatheter aortic valve replacement: A meta-analysis. Catheter Cardiovasc Interv. 2018;91(2):330-342. doi:10.1002/ccd.27207

7. WOOD DA, LAUCK SB, CAIRNS JA, et al. The Vancouver 3M (Multidisciplinary, Multimodality, But Minimalist) Clinical Pathway Facilitates Safe Next-Day Discharge Home at Low-, Medium-, and High-Volume Transfemoral Transcatheter Aortic Valve Replacement Centers: The 3M TAVR Study. JACC Cardiovasc Interv. 2019;12(5):459-469.

8. ICHIBORI Y, LI J, PATEL T, et al. Short-Term and Long-Term Outcomes of Patients Undergoing Urgent Transcatheter Aortic Valve Replacement Under a Minimalist Strategy. J Invasive Cardiol. 2019;31(2):30-36.

9. YASUHIRO ICHIBORI, JUN LI, ANGELA DAVIS; TORAL M. PATEL, JERRY LIPINSKI ; MUHAMMAD PANHWAR, MD1, 2; PETAR SARIC, MD1, 2; GHAZANFAR QURESHI, MD1 2; Feasibility and Safety of Adopting Next-Day Discharge as First-Line Option After Transfemoral Transcatheter Aortic Valve Replacement. J Invasive Cardiol. 2019;31(3):64-72.

10. DE BRITO FS, CARVALHO LA, SARMENTO-LEITE R, et al. Outcomes and predictors of mortality after transcatheter aortic valve implantation: Results of the Brazilian registry. Catheter Cardiovasc Interv. 2015;85(5):E153-62.

11. MAYR NP, WIESNER G, HUSSER O, et al. Critical adverse 
events during transfemoral TAVR in conscious sedation. Is an anesthesiologic support mandatory? Cardiovasc Revascularization Med. 2018;19(6s):41-46.

12. THYREGOD HGH, STEINBRÜCHEL DA, IHLEMANN N, et al. Transcatheter versus surgical aortic valve replacement in patients with severe aortic valve stenosis: 1-year results from the all-comers NOTION randomized clinical trial. J Am Coll Cardiol. 2015;65(20):2184-2194.

13. LEON MB, SMITH CR, MACK MJ, et al. Transcatheter or Surgical Aortic-Valve Replacement in Intermediate-Risk Patients. N Engl J Med. 2016;374:1609-1620.

14. CHANDRASEKHAR J, DANGAS G, YU J, et al. Sex-Based Differences in Outcomes With Transcatheter Aortic Valve Therapy: TVT Registry From 2011 to 2014. J Am Coll Cardiol. 2016;68(25):2733-2744.
15. YOUSIF N, OBEID S, BINDER R , DENEGRI A SM. Impact of gender on outcomes after transcatheter aortic valve implantation (TAVI). J Geriatr Cardiol. 2018;15(6):394-400.

16. ITZHAKI BEN ZADOK O, KORNOWSKI R, FINKELSTEIN A, et al. Temporal Trends in Gender-Related Differences and Outcomes in Patients Who Underwent Transcatheter Aortic Valve Implantation (from the Israeli Transcatheter Aortic Valve Implantation Multicenter Registry). Am J Cardiol. 2019;123(1):108-115.

17. A. PINEDA, J. HARRISON, N. KLEIMAN, C. RIHAL NB. Incidence and outcomes of surgical bailout during TAVR. JACC Cardiovasc Interv. 2019;12(18):1751-1764.

18. MALUENDA G, SEPÚlVEDA E, ALFARO M, et al. Costo-Efectividad de Reemplazo Valvular Aórtico Percutáneo vs Terapia Conservadora en la Estenosis Aórtica de muy alto riesgo en un centro terciario chileno. Rev Chil Cardiol. 2015;15:11-17. 